\title{
Long-term survival rates of patients with nasopharyngeal carcinoma treated by radiochemotherapy: a retrospective cohort study
}

\author{
Ali Kazemian ${ }^{1,2}$, Reza Ghalehtaki ${ }^{1,2^{*}}$, Borna Farazmand ${ }^{1}$, Maryam Taherioun ${ }^{2}$, Mahdieh Razmkhah², \\ Nastaran Khalili ${ }^{3,4}$, Farshid Farhan ${ }^{2}$, Mahdi Aghili ${ }^{1,2}$ and Ebrahim Esmati ${ }^{2}$
}

\begin{abstract}
Background: Nasopharyngeal cancer (NPC) is showing an increasing incidence in Iran. Radiation is the main treatment of this cancer. Use of new techniques such as intensity-modulated radiotherapy (IMRT) is on the rise. Here, we aimed to evaluate the oncological outcomes of NPC patients treated with three-dimensional conformal radiotherapy (3DCRT) for a more reliable comparison with IMRT in the future.

Results: We reviewed the medical records of 106 patients with NPC treated by definitive radiotherapy from 2007 to 2016. Patients were treated with $70 \mathrm{~Gy}$ in 2-Gy fractions. Twenty-one patients died during the follow-up period. Twenty-nine patients suffered from locoregional or distant recurrences. Of these, 6 recurred after 2 years of treatment completion. The 2-year and 5-year overall survival rates were $81 \%$ and $76 \%$, respectively. The 2-year and 5-year progression-free survival rates were $72 \%$ and $63 \%$, respectively. The 5 -year locoregional recurrence and distant metastasis-free survival rates were $68 \%$ and $69 \%$, respectively.

Conclusion: Due to high survival rates of NPC and the importance of receiving planned total dose of RT, the treatment-related toxicity and quality of life are critical considerations both for patients during active treatment and for survivors.
\end{abstract}

Keywords: Chemoradiation, Head and neck cancer, Iran, Nasopharyngeal cancer, Conformal radiotherapy

\section{Background}

Nasopharyngeal cancers or NPCs are among the rarest malignancies worldwide with a certain geographic distribution. NPC is endemic in areas such as Southern China, Northern Africa, Southeast Asia, and the Arctic. According to the few present studies regarding NPC incidence among the Iranian population, it is estimated that its incidence rate is 0.38 per 100,000 . This places Iran among

\footnotetext{
*Correspondence: rghalehtaki@tums.ac.ir

${ }^{2}$ Department of Radiation Oncology, Cancer Institute, Imam Khomeini Hospital Complex, Tehran University of Medical Sciences, Tehran, Iran Full list of author information is available at the end of the article
}

relatively high prevalent areas, although not endemic [1]. Known risk factors of NPC include EBV infection and consumption of salt-preserved foods, low intake of fruits and vegetables, tobacco smoking, and male sex. This disease also has a strong genetic-related pattern, as it showed a significantly higher incidence rate among Chinese people born in western countries [2].

This cancer is usually diagnosed late at advanced stages (stages III and IV with or without distant metastases), most probably because of the anatomical site of the origin. Due to limited and difficult surgical access to the nasopharynx, the primary treatment of NPC is by delivering therapeutic radiation. Early stages (T1N0) of 
NPC are treated by radiotherapy alone. However, locally advanced disease is treated by combined chemoradiotherapy [3]. The prognosis in the early stages is desirable. In contrast, for locally-advanced stages, the 5-year overall survival (OS) rates are estimated to be $53-80 \%$ and $28-61 \%$ in stages III and IV, respectively [4].

There are unsolved challenges in the treatment of NPC. Selecting the best technique for radiation, consumption of induction or adjuvant chemotherapy, the sequence of administration of cisplatin, as well as finding the best practice for the management of recurrent cases are subjects that experts have yet to reach a consensus upon [5, 6].

Although NPC is not considered endemic in Iran, it has shown an increasing pattern of incidence among the Iranian population. In addition, we do not have reliable information of the long-term outcome of Iranian patients who have been treated by the latest available approach. As long as our knowledge about this issue remains obscure, taking further steps to improve the disease outcome is implausible. As a result, we performed this study to evaluate overall survival (OS) and disease-free survival (DFS) rates among Iranian patients with NPC and determine its correlation with treatment characteristics.

\section{Methods}

\section{Patients and study design}

In this study, we retrospectively reviewed the medical records of 106 patients with biopsy-proven nasopharyngeal cancer. They were all treated by definitive radiotherapy from 2007 to 2016 in the radiation oncology ward of Cancer Institute of Iran, Tehran, Iran. The exclusion criteria consisted of distant nodal, visceral, or bone metastasis, recurrent disease, and also positive history of prior radiotherapy or chemotherapy for other head and neck malignancies. Our institutional review board and ethics committee approved this study (ethics code: IR.TUMS. IKHC.REC.1399.102 available at ethics.research.ac.ir) in agreement with the World Medical Association Declaration of Helsinki "Ethical Principles for Medical Research Involving Human Subjects," amended in October 2013, (www.wma.net). Patients were made aware that their disease information would be used for medical research purposes only after de-identification of personal information. Written informed consent was obtained from all patients at first admission prior to enrollment in the study.

\section{Clinical staging definition and treatment design}

According to the 7th edition of American Joint Committee on Cancer guideline for nasopharyngeal cancer, staging was done based on medical history, physical examination, chest X-ray (CXR) findings, laboratory data, and nasal endoscopy as well as magnetic resonance imaging (MRI) of neck and skull base [7]. Additional tests were indicated in case of uncertainty of diagnosis after initial evaluation. For instance, if the patients had N3 involvement (a supraclavicular or larger than $6 \mathrm{~cm}$ node), they underwent pan computed tomography $(\mathrm{CT})$ and whole-body bone scan. For tumors with non-keratinized undifferentiated histology, immunohistochemistry was performed to rule out lymphoma or possible diagnoses other than carcinoma.

Patient immobilization, simulation, and treatment planning were performed according to our department protocol for 3-dimensional (3D) conformal radiotherapy. The radiation oncologists (RO) delineated the gross tumor volume (GTV) and clinical target volume (CTV) based on information from nasal endoscopy, MRI, and physical exam findings. The target delineation was in accordance with the protocol of RTOG 0615 [8]. The added geometrical margin to conform the planning target volume (PTV) was $0-1 \mathrm{~cm}$, based on proximity to critical structures. Patients were treated with 70 Gy in 2-Gy fractions, five fractions per week in 7 weeks. The treatment was mainly carried out by 6-18 MV external beam photons. The exclusive 10-Gy dose for the GTV was delivered by an anterior and two 6-MV lateral beams or just two 18-MV lateral beams. For boosting the dose to posterior neck nodes, we utilized 9-12 MeV electron beams based on the depth of nodes and their proximity to the spinal cord. The application of brachytherapy for boosting the GTV was at the discretion of the radiation oncologist. In these cases, two 7-Gy fractions of high dose rate (HDR) brachytherapy were performed with a 1-week interval.

Patients with tumor staged as T2 or N1 and higher received concomitant chemotherapy (ChT) with radiation. The routine ChT regimen was weekly cisplatin (30-35 $\left.\mathrm{mg} / \mathrm{m}^{2}\right)$; however, 3-weekly cisplatin was occasionally used as well. Several of the attending physicians used off-label cetuximab for patients who did not tolerate cisplatin. The administration of induction or adjuvant ChT was at the discretion of the consultant radiation oncologist.

\section{Follow-up and recurrence}

Patients were visited weekly during radiotherapy. Patients disclosed their new complaints and the RO carried out relevant physical examination or laboratory tests if needed. Follow-up visit was 1 month after the completion of treatment, then, every 3 months within the first 2 years, every 6 months for years 3-5, and annually thereafter. The follow-up visit consisted of history, physical exam, and nasal endoscopy for all patients and MRI if 
indicated. Additional diagnostic tests including CT scan of the neck, thorax, and abdomen, as well as whole body bone scan, were demanded based on clinical judgment.

In cases suspicious of recurrence, biopsy was mandatory for confirmation before starting the appropriate treatment. Treatment options for local recurrence included surgical resection, re-irradiation, and/or brachytherapy. For regional recurrence in the neck, neck dissection with/without postoperative radiation was the main option. For distant recurrence, systemic therapy and palliative radiation were the recommended options.

\section{Statistical analysis}

We recruited all eligible patients, consecutively, without sample size and power calculation. The Statistical Package for Social Sciences software (SPSS version 20.0, IBM Corporation, Chicago, IL, US) was opted to analyze data. To describe the data we used frequency with percent and median with inter-quartile range (IQR). Kaplan-Meyer survival analysis was applied for calculating overall survival (OS) and progression-free survival (PFS) rate. The OS was calculated from initiation of radiation therapy to the date of death or last follow-up visit. For PFS, the end-point was the occurrence of locoregional or distant metastasis or death. These rates were presented with 95\% confidence interval (CI). Cox proportional hazards test and log-rank tests were used to determine any significant predictors of OS and PFS. Level of significance was considered $p<0.05$.

\section{Results}

\section{Patients' characteristics}

One-hundred and six patients with newly diagnosed non-metastatic NPC were included in the analysis. The median age of patients was 49 years (IQR: $40-59$ ). The youngest and oldest patients were 14 and 78 years old, respectively. Thirty patients were female $(28.3 \%)$. Regarding the histopathology of specimens, $88.7 \%, 2.8 \%$, and $7.5 \%$ were undifferentiated, keratinizing, and non-keratinizing NPC, respectively. In one patient, the histology was unspecified. The available detailed $\mathrm{T}$ and $\mathrm{N}$ status of 104 patients is shown in Table 1 . However, a specific clinical stage was defined for all patients. Nine (8.5\%), 23 (21.7\%), 46 (43.4\%), 12 (11.3\%), and 16 (15.1\%) patients were classified as stage I, II, III, IVa, and IVb, respectively.

\section{Treatment characteristics}

Patients underwent radiotherapy with a median dose of 70 Gy (IQR: 70-70). Four patients received intracavitary brachytherapy as the boost to GTV (12-16.5 Gy in 2-3 fractions). Median external radiotherapy duration was 55 days (IQR: 50-61 days). Due to delays within treatment
Table $1 \mathrm{~T}$ and $\mathrm{N}$ characteristics of the nasopharyngeal tumors $(n=104)$

\begin{tabular}{llllll}
\hline \multirow{2}{*}{$\begin{array}{l}\text { Tumor } \\
\text { size }\end{array}$} & \multicolumn{5}{l}{ Regional lymph node involvement $(\boldsymbol{n}, \mathbf{\%})$} \\
\cline { 2 - 6 } & N0 & N1 & N2 & N3 & Total \\
\hline T1 & $9(8.7 \%)$ & $9(8.7 \%)$ & $15(14.4 \%)$ & $2(1.9 \%)$ & $\mathbf{3 5 ( 3 3 . 7 \% )}$ \\
T2 & $5(4.8 \%)$ & $7(6.7 \%)$ & $12(11.5 \%)$ & $8(7.7 \%)$ & $\mathbf{3 2}(\mathbf{3 0 . 8} \%)$ \\
T3 & $7(6.7 \%)$ & $10(9.6 \%)$ & $2(1.9 \%)$ & $3(2.9 \%)$ & $\mathbf{2 2 ( 2 1 . 2 \% )}$ \\
T4 & $1(1 \%)$ & $4(3.8 \%)$ & $7(6.7 \%)$ & $3(2.9 \%)$ & $\mathbf{1 5 ( 1 4 . 4 \% )}$ \\
Total & $\mathbf{4 ( 1 2 . 1 \% )}$ & $\mathbf{1 2}$ & $\mathbf{1 3}$ & $\mathbf{4 ( 1 2 . 1 \% )}$ & \\
& & $\mathbf{( 3 6 . 4 \% )}$ & $\mathbf{( 3 9 . 4 \% )}$ & & \\
\hline
\end{tabular}

time, $77.5 \%$ of patients completed their external radiation therapy after 7 weeks.

Fifty-two patients $(49.1 \%)$ received induction ChT. The most common regimen was docetaxel plus cisplatin plus 5-flourouracil (also known as TPF regimen) administered to $35.6 \%$ of patients followed by cisplatin plus 5 -flourouracil (a.k.a PF regimen) in $10.6 \%$ of patients. Ninety-eight patients $(92.5 \%)$ received concurrent ChT. Concurrent regimens were weekly cisplatin $(59.4 \%)$ followed by 3-weekly cisplatin (31.1\%) and off-label cetuximab (1.9\%). Regarding concurrent chemotherapy, 61.7\% and $96.9 \%$ of patients received the minimum sufficient dose of weekly (at least 6 cycles) and 3-weekly (at least 2 cycles) cisplatin, respectively. Administration of induction ChT was not significantly associated with the ability to receive a minimum sufficient dose of weekly cisplatin (64.5\% versus $58.6 \%$ in patients with and without induction ChT, respectively, $p=0.791)$. Only six patients $(5.7 \%)$ received consolidative (post-radiation) ChT.

\section{Disease outcomes}

The median follow-up time in our cohort was 48 months (95\% CI $=37.7-58.3$ ). Twenty-one patients died during the follow-up period. During the same period, 29 patients suffered from locoregional or distant recurrences. In 6 (20.7\%) of these, the disease recurred after 2 years of primary treatment termination that is called a "late recurrence." The most common site of metastasis was the liver followed by bone and lungs $(16.7 \%, 12.5 \%$, and $8.3 \%$ of total patients, respectively).

The 2-year, 5-year, and 8-year OS rates were $81 \%$ (95\% $\mathrm{CI}=73-89), 76 \%(95 \% \mathrm{CI}=66-86)$, and $74 \%(95 \% \mathrm{CI}=$ 64-84), respectively (Fig. 1). Regarding the stage at diagnosis, the 5 -year OS rates were $88 \%$ for stage I, $84 \%$ for stage II, $73 \%$ for stage III, and $72 \%$ for stage IV.

The 2-year, 5-year, and 8-year PFS rates were 72\% (95\% $\mathrm{CI}=62-82), 63 \%(95 \% \mathrm{CI}=53-73)$, and $63 \%(95 \% \mathrm{CI}$ =53-73), respectively (Fig. 2). The 5-year locoregional recurrence and distant metastasis-free survival rates 


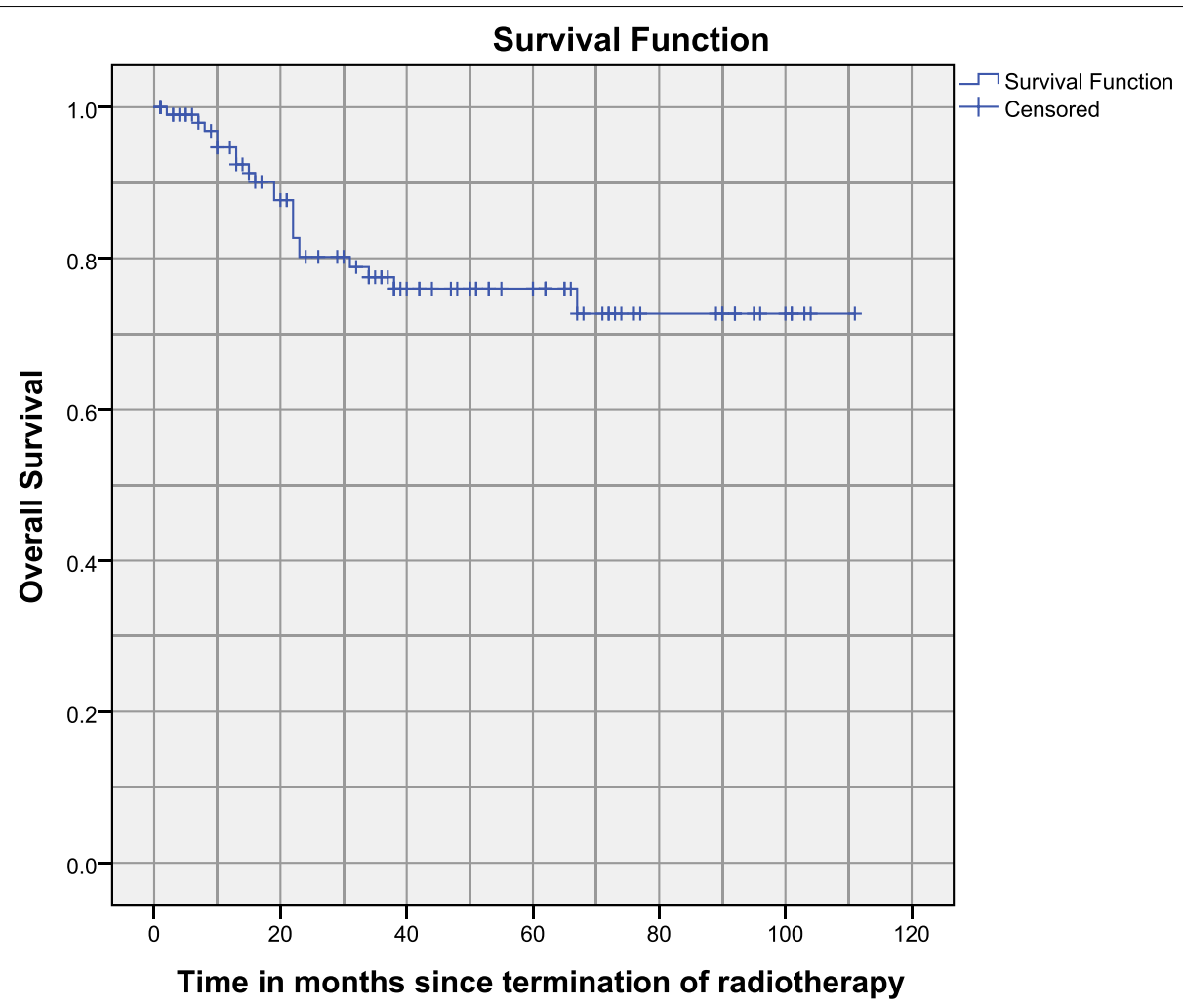

Fig. 1 Kaplan-Meyer survival curve for overall survival

were $68 \%(95 \% \mathrm{CI}=58-78)$ and $69 \%(95 \% \mathrm{CI}=59-79)$, respectively.

\section{Predictive variables of survival}

Results of the multivariate Cox proportional hazard test for determining predictors of overall and disease-free survival are shown in Table 2. Statistically significant predictors for OS were distant metastasis, locoregional recurrence, and receiving enough cycles of concurrent ChT. In patients who received the total planned RT dose (at least $70 \mathrm{~Gy}$ ), a trend for improved OS was observed ( $P$ $<0.1)$. However, receiving the total planned RT dose was the single significant predictor for PFS (Table 3).

\section{Discussion}

The management of nasopharyngeal carcinoma poses a challenge due to its difficult anatomic accessibility. This site is not easily surgically accessible, so radiation therapy is considered the primary treatment. With advances in radiation planning and treatment delivery, possible radiation-associated toxicities have decreased substantially. Nowadays, intensity-modulated radiotherapy (IMRT) is considered the standard of choice for treating NPC [9]. However, this relatively expensive technique is not performed routinely in developing countries and less economically advantaged parts of the world. In order to evaluate and compare the efficacy of the IMRT technique, first, we should be informed of the treatment outcomes of older techniques such as conventional (twodimensional or 2D) and three-dimensional conformal radiotherapy (3DCRT). In this study, we reported the long-term outcomes of treating NPC with 3D-CRT in our institution before moving forward to IMRT in the near future.

The rate of OS in our study was in agreement with other studies employing 3DCRT or IMRT [10]. The major contribution of conformal techniques to the overall survival is around $8 \%$ in locoregional control and OS compared to the 2D era [2]. Moving from 3D to IMRT has not impacted the rate of oncological outcomes significantly. The main reason is that the total dose is still the same with both techniques. However, the rate of disturbing side-effects, namely xerostomia, has decreased substantially with IMRT. In fact, this is why IMRT is the preferred technique for the treatment of NPC [11]. Regarding the high cure rate of patients with nasopharyngeal cancer, the quality of life of survivors is of outmost importance. This justifies the emphasis on IMRT for the treatment of nasopharyngeal carcinoma. 


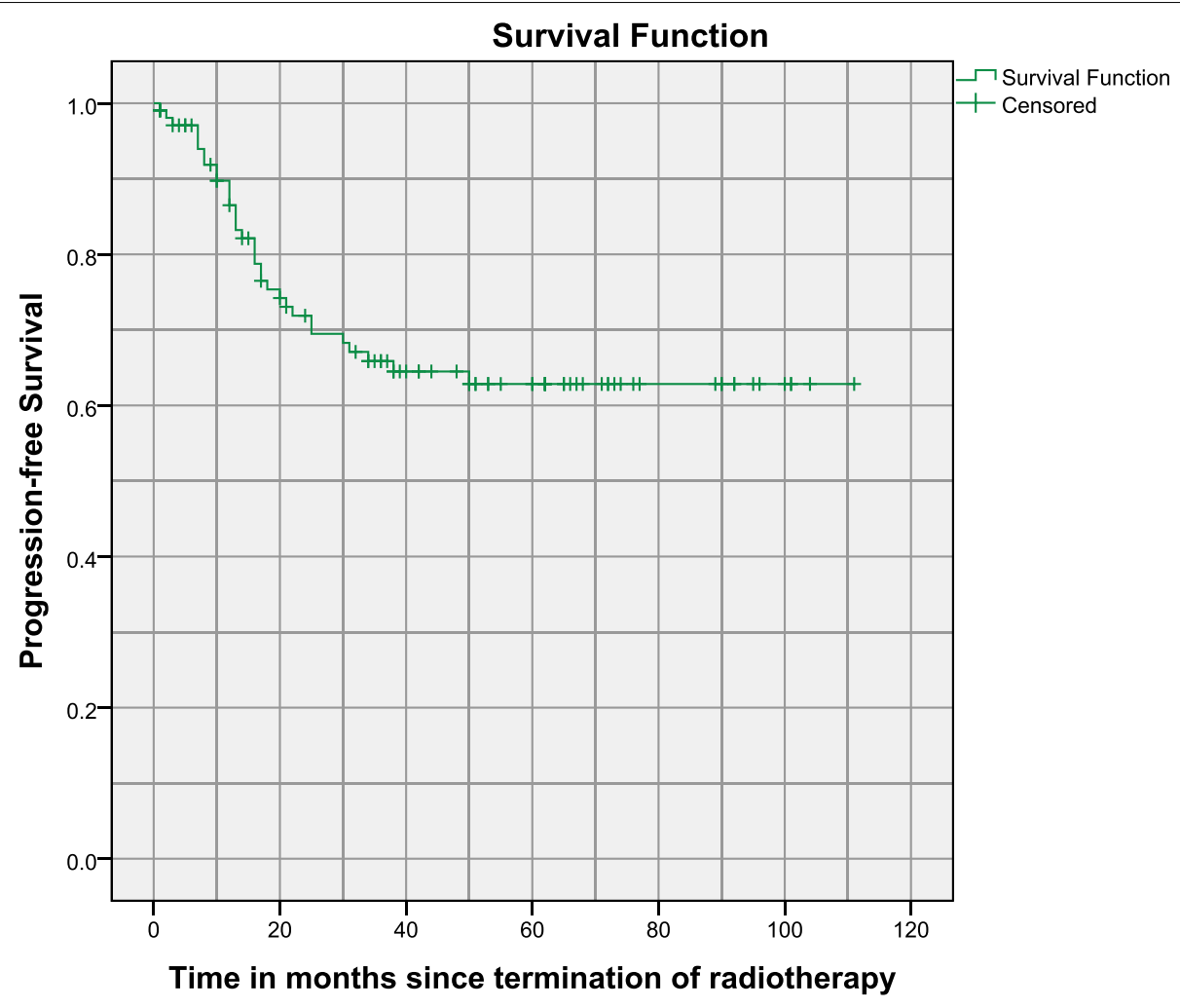

Fig. 2 Kaplan-Meyer survival curve for progression free survival

Table 2 Predictors of progression free survival

\begin{tabular}{|c|c|c|c|c|}
\hline & \multirow[t]{2}{*}{$P$ value } & \multirow[t]{2}{*}{$\operatorname{Exp}(B)$} & \multicolumn{2}{|c|}{$\begin{array}{l}95.0 \% \mathrm{Cl} \text { for } \\
\operatorname{Exp(B)}\end{array}$} \\
\hline & & & Lower & Upper \\
\hline Age & .125 & 1.030 & .992 & 1.069 \\
\hline Sex & .078 & 2.838 & .890 & 9.050 \\
\hline \multicolumn{5}{|l|}{ T stage } \\
\hline T1 (reference) & .270 & & & \\
\hline $\mathrm{T} 2$ & .232 & .475 & .141 & 1.607 \\
\hline T3 & .054 & .321 & .101 & 1.018 \\
\hline $\mathrm{T} 4$ & .171 & .395 & .105 & 1.491 \\
\hline \multicolumn{5}{|l|}{ N stage } \\
\hline N0 (reference) & .392 & & & \\
\hline N1 & .111 & 4.489 & .708 & 28.459 \\
\hline N2 & .189 & 3.071 & .576 & 16.381 \\
\hline N3 & .119 & 3.597 & .720 & 17.962 \\
\hline Induction $\mathrm{ChT}^{\mathrm{a}}$ & .312 & .616 & .241 & 1.577 \\
\hline Receiving Planned RT dose (70Gy) & .041 & 2.998 & 1.044 & 8.609 \\
\hline Receiving Planned ChT Cycles ${ }^{b}$ & .251 & 1.785 & .663 & 4.805 \\
\hline Duration of RT & .121 & .966 & .926 & 1.009 \\
\hline
\end{tabular}

${ }^{\text {a Chemotherapy }}$

${ }^{\mathrm{b}}$ Two 3-weekly or 6-weekly cisplatin
It is well established that NPC is more sensitive to radiation in comparison to head and neck squamous cell carcinoma located in other sites [12]. In our study, there was a trend for better survival in favor of receiving the planned RT dose of 70 Gy compared to fewer doses. However, the duration of RT was not related to OS. Our results were consistent with previous studies indicating that the total dose is more important than the duration of the dose. Numerous investigations have been performed to accelerate the RT schedule in order to achieve better results but similar conclusions have not been reached [2]. However, simultaneous integrated boost is a popular technique for IMRT since some studies showed that accelerated radiotherapy is associated with better locoregional control [13].

Our study showed the contribution of receiving the planned dose of concurrent ChT on the improvement of OS. The hallmark Intergroup (INT) 0099 phase III trial which established the chemoradiotherapy for NPC as the standard treatment, employed 3 cycles of concurrent cisplatin and 2 cycles of consolidative PF in the study protocol [14]. Exploratory analysis showed that the benefit of concurrent ChT in increasing OS compared with radiotherapy alone, was restricted to whom received at least two concurrent cycles equal to the $200 \mathrm{mg} / \mathrm{m}^{2}$ cisplatin [15]. 
Table 3 Predictors of overall survival (Cox-hazard test)

\begin{tabular}{|c|c|c|c|c|}
\hline & \multirow[t]{2}{*}{$P$ value } & \multirow[t]{2}{*}{$\operatorname{Exp}(B)$} & \multicolumn{2}{|c|}{$\begin{array}{l}95.0 \% \mathrm{Cl} \text { for } \\
\operatorname{Exp}(\mathrm{B})\end{array}$} \\
\hline & & & Lower & Upper \\
\hline Age & .195 & 1.038 & .981 & 1.099 \\
\hline Sex & .400 & 1.963 & .408 & 9.448 \\
\hline \multicolumn{5}{|l|}{ T Stage } \\
\hline T1 (reference) & .155 & & & \\
\hline $\mathrm{T} 2$ & .522 & 1.883 & .271 & 13.065 \\
\hline T3 & .115 & .247 & .044 & 1.405 \\
\hline T4 & .850 & 1.215 & .161 & 9.185 \\
\hline \multicolumn{5}{|l|}{ N Stage } \\
\hline N0 (reference) & .445 & & & \\
\hline N1 & .170 & .162 & .012 & 2.177 \\
\hline N2 & .223 & .228 & .021 & 2.456 \\
\hline N3 & .837 & .821 & .125 & 5.386 \\
\hline Induction $\mathrm{ChT}^{\mathrm{a}}$ & .463 & .625 & .178 & 2.191 \\
\hline Receiving Planned RT dose (70Gy) & .093 & 3.675 & .805 & 16.772 \\
\hline Receiving Planned ChT Cycles ${ }^{b}$ & .025 & 6.351 & 1.268 & 31.815 \\
\hline Duration of RT & .831 & 1.007 & .948 & 1.069 \\
\hline Locoregional recurrence & .025 & .157 & .031 & .796 \\
\hline Distant metastasis & .000 & .014 & .002 & .107 \\
\hline
\end{tabular}

${ }^{a}$ Chemotherapy

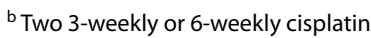

We found that the administration of induction or consolidative ChT was not associated with better survival. In the INT 0099 trial, only about half of the patients received all two adjuvant cycles. Thus, many physicians became doubtful of the efficacy of consolidation ChT after concurrent chemoradiation [15, 16]. Even Al-Sarraf himself proposed the reversal of the chemotherapy plan before chemoradiation for better tolerance and better outcomes [3]. Some researchers believe this setting could impact failure-free survival by decreasing the rate of distant metastases [17-19]. But owing to the inconsistency in data regarding OS benefit with induction ChT, this has not been a standard general approach yet. However, induction ChT seems to be an acceptable approach for bulky, especially N2-3, locally advanced disease [20,21]. In summary, the standard treatment still remains to be concurrent chemoradiotherapy.

The main strength of our study was the long-term follow-up of patients. For the nasopharyngeal carcinoma, as a curable head and neck malignancy, short-term followup could not precisely assess the oncological outcomes. In addition, there is the possibility of patients with late locoregional recurrences that are still eligible candidates for curative treatment and have good chance of survival.

Our study had some limitations. Although one of the most important differences between IMRT and 3DCRT is the lower rate of toxicities, due to the retrospective nature of this study, we could not assess acute and late treatment-related toxicities. Another limitation was the inability to control treatment portals in our institution. Due to the high volume of patients being treated in our center, it is not feasible to control the portals with port films. Moreover, our apparatuses lack an electronic portal imaging device or cone-beam CT. Furthermore, the regimen of concurrent chemotherapy was at the discretion of the attending radiation oncologist. So, different regimens were opted based on the physician's preference. These various regimens could possibly lead to different outcomes.

\section{Conclusion}

The long-term outcome of patients with nasopharyngeal carcinoma treated with 3DCRT in our center was similar to previously published studies applying conformal techniques such as 3DCRT and IMRT. Due to high survival rates of NPC and the importance of receiving planned total dose of RT, the treatment-related toxicity and quality of life are critical considerations both for patients during active treatment and for survivors. We emphasize on implementing IMRT as soon as possible for nasopharyngeal carcinoma regarding the lower rate of toxicity and better quality of life it offers.

\section{Abbreviations \\ NPC: Nasopharyngeal cancer; EBV: Epstein-Barr virus; IMRT: Intensity- modulated radiation therapy; 3DCRT: 3-dimensional conformal radiation therapy; 2D: 2-dimensional; OS: Overall survival; DFS: Disease-free survival; PFS: Progressive-free survival; CXR: Chest X-ray; MRI: Magnetic resonance imaging; CT: Computed tomography; RO: Radiation oncologist; GTV: Gross tumor volume; CTV: Clinical tumor volume; PTV: Planning tumor volume; HDR: High dose rate; $\mathrm{ChT}$ : Chemotherapy.}

\section{Acknowledgements}

We thank our colleagues from the department of Radiation Oncology of Cancer Institute, Imam Khomeini Hospital Complex for their contribution to patients' accrual and enrollment. We are deeply grateful to our beloved clerks in Medical Records Archive who helped us a lot during data collection.

\section{Authors' contributions}

AK: designing the study, collecting, analyzing and interpreting the data and making the decision to submit for publication. RG: designing the study, collecting, analyzing and interpreting the data, writing the report and making the decision to submit for publication. BF: designing the study, collecting, analyzing and interpreting the data. MT: writing the report. MR: writing the report. NK: collecting, analyzing and interpreting the data. FF: collecting, analyzing and interpreting the data. MA: collecting, analyzing and interpreting the data. EE: collecting, analyzing and interpreting the data. The authors read and approved the final manuscript.

\section{Funding}

This study was supported and funded by the Tehran University of Medical Sciences (TUMS) With Grant No: 99-1-248-47949.

Availability of data and materials Not applicable 


\section{Declarations}

\section{Ethics approval and consent to participate:}

This study was reviewed and approved by the ethics committee of Tehran University of Medical Sciences with code: IR.TUMS.IKHC.REC.1399.102 (accessible at: ethics.research.ac.ir). Informed written consent to participate in the study was provided by all participants (or their parent or legal guardian in the case of children under 16) at the time of admission to the radiation oncology department.

\section{Consent for publication}

Not applicable

\section{Competing interests}

The authors declare that they have no competing interests. All of the authors declare no industrial link

\section{Author details}

${ }^{1}$ Radiation Oncology Research Center, Cancer Research Institute, Tehran University of Medical Sciences, Tehran, Iran. ${ }^{2}$ Department of Radiation Oncology, Cancer Institute, Imam Khomeini Hospital Complex, Tehran University of Medical Sciences, Tehran, Iran. ${ }^{3}$ School of Medicine, Tehran University of Medical Sciences, Tehran, Iran. ${ }^{4}$ Cancer Immunology Project, Universal Scientific Education and Research Network (USERN), Tehran, Iran.

\section{Received: 5 December 2021 Accepted: 17 January 2022}

Published online: 10 February 2022

\section{References}

1. Safavi A, Raad N, Raad N, Ghorbani J (2015) Epidemiology of nasopharyngeal cancers in Iran: a 6-year report. Asian Pac J Cancer Prev 16:44474450. https://doi.org/10.7314/APJCP.2015.16.10.4447

2. Chua MLK, Wee JTS, Hui EP, Chan ATC (2016) Nasopharyngeal carcinoma. In: The lancet. Lancet Publishing Group, pp 1012-1024

3. Chan ATC (2010) Nasopharyngeal carcinoma. In: Annals of oncology. Curr Treat Options Oncol, pp 21-32

4. Fang FM, Chien CY, Tsai WL, Chen HC, Hsu HC, Lui CC, Huang TL, Huang HY (2008) Quality of life and survival outcome for patients with nasopharyngeal carcinoma receiving three-dimensional conformal radiotherapy vs. intensity-modulated radiotherapy-a longitudinal study. Int J Radiat Oncol Biol Phys 72:356-364. https://doi.org/10.1016/j.jirobp. 2007.12.054

5. Qu S, Liang ZG, Zhu XD (2015) Advances and challenges in intensitymodulated radiotherapy for nasopharyngeal carcinoma. Asian Pac J Cancer Prev 16:1687-1692

6. Xu T, Tang J, Gu M, et al. Oncology HY-C, et al. Recurrent nasopharyngeal carcinoma: a clinical dilemma and challenge. Available from: https:// www.ncbi.nlm.nih.gov/pmc/articles/PMC3805410. [Accessed 2020 Feb 4]

7. Edge SB, Compton CC (2010) The American joint committee on cancer: the 7th edition of the AJCC cancer staging manual and the future of TNM. Ann Surg Oncol 17:1471-1474

8. Lee NY, Zhang Q, Pfister DG, Kim J, Garden AS, Mechalakos J, Hu K, Le QT, Colevas AD, Glisson BS, Chan ATC, Ang KK (2012) Addition of bevacizumab to standard chemoradiation for locoregionally advanced nasopharyngeal carcinoma (RTOG 0615): a phase 2 multi-institutional trial. Lancet Oncol 13:172-180. https://doi.org/10.1016/S1470-2045(11) 70303-5

9. Takiar V, Ma D, Garden AS, Li J, Rosenthal DI, Beadle BM, Frank SJ, Fuller CD, Gunn GB, Morrison WH, Hutcheson K, El-Naggar AK, Gold KA, Kupferman ME, Phan J (2016) Disease control and toxicity outcomes for T4 carcinoma of the nasopharynx treated with intensity-modulated radiotherapy. Head Neck 38(Suppl 1):E925-E933. https://doi.org/10.1002/hed.24128

10. Slevin F, Pan S, Mistry H, Sen M, Foran B, Slevin N, Dixon L, Thomson D, Prestwich R (2019) A multicentre UK study of outcomes of nasopharyngeal carcinoma treated with intensity-modulated radiotherapy \pm chemo therapy. Clin Oncol 32:238-249. https://doi.org/10.1016/j.clon.2019.11. 009
11. Fang F-M, Chien C-Y, Tsai W-L, Chen H-C, Hsu H-C, Lui C-C, Huang T-L, Huang H-Y (2008) Quality of life and survival outcome for patients with nasopharyngeal carcinoma receiving three-dimensional conformal radiotherapy vs. intensity-modulated radiotherapy-a longitudinal study. Int J Radiat Oncol Biol Phys 72:356-364. https://doi.org/10.1016/j.jirobp. 2007.12.054

12. Barnett GC, West CML, Dunning AM, Elliott RM, Coles CE, Pharoah PDP, Burnet NG (2009) Normal tissue reactions to radiotherapy: towards tailoring treatment dose by genotype. Nat Rev Cancer 9:134-142

13. Wolden SL, Chen WC, Pfister DG, Kraus DH, Berry SL, Zelefsky MJ (2006) Intensity-modulated radiation therapy (IMRT) for nasopharynx cancer: update of the memorial Sloan-Kettering experience. In: International journal of radiation oncology biology physics. Elsevier, pp 57-62

14. Al-Sarraf M, LeBlanc M, Giri PGS, Fu KK, Cooper J, Vuong T, Forastiere AA, Adams G, Sakr WA, Schuller DE, Ensley JF (1998) Chemoradiotherapy versus radiotherapy in patients with advanced nasopharyngeal cancer: phase III randomized intergroup study 0099. J Clin Oncol 16:1310-1317. https://doi.org/10.1200/JCO.1998.16.4.1310

15. Chen L, Hu CS, Chen XZ, Hu GQ, Bin CZ, Sun Y, Li WX, Chen YY, Xie FY, Liang SB, Chen Y, Xu TT, Li B, Long GX, Wang SY, Zheng BM, Guo Y, Sun Y, Mao YP, Tang LL, Chen YM, Liu MZ, Ma J (2012) Concurrent chemoradiotherapy plus adjuvant chemotherapy versus concurrent chemoradiotherapy alone in patients with locoregionally advanced nasopharyngeal carcinoma: a phase 3 multicentre randomised controlled trial. Lancet Oncol 13:163-171. https://doi.org/10.1016/S1470-2045(11)70320-5

16. Yang S, Lin S, Fu Q, Cai B, Kong F, Huang G, Li F, Wang H (2015) The effect of adjuvant chemotherapy on survival in patients with residual nasopharyngeal carcinoma after undergoing concurrent chemoradiotherapy. PLoS One 10. https://doi.org/10.1371/journal.pone.0120019

17. Lee AWM, Lau KY, Hung WM, Ng WT, Lee MCH, Choi CW, Chan CCC, Tung R, Cheng PTC, Yau TK (2008) Potential improvement of tumor control probability by induction chemotherapy for advanced nasopharyngeal carcinoma. Radiother Oncol 87:204-210. https://doi.org/10.1016/j.radonc. 2008.02.003

18. Sun Y, Li WF, Chen NY, Zhang N, Hu GQ, Xie FY, Sun Y, Chen XZ, Li JG, Zhu XD, Hu CS, Xu XY, Chen YY, Hu WH, Guo L, Mo HY, Chen L, Mao YP, Sun R, Ai P, Liang SB, Long GX, Zheng BM, Feng XL, Gong XC, Li L, Shen CY, Xu JY, Guo Y, Chen YM, Zhang F, Lin L, Tang LL, Liu MZ, Ma J (2016) Induction chemotherapy plus concurrent chemoradiotherapy versus concurrent chemoradiotherapy alone in locoregionally advanced nasopharyngeal carcinoma: a phase 3, multicentre, randomised controlled trial. Lancet Oncol 17:1509-1520. https://doi.org/10.1016/S1470-2045(16)30410-7

19. Le QT, Colevas AD, O'Sullivan B, Lee AWM, Lee N, Ma B, Siu LL, Waldron J, Lim CM, Riaz N, Lynn J, Malik S (2019) Current treatment landscape of nasopharyngeal carcinoma and potential trials evaluating the value of immunotherapy. J Natl Cancer Inst 111:655-663

20. Kawahira M, Yokota T, Hamauchi S, Onozawa Y, Ogawa H, Onoe T, Kamijo T, lida Y, Nishimura T, Onitsuka T, Yasui H (2017) Survival benefit of adding docetaxel, cisplatin, and 5-fluorouracil induction chemotherapy to concurrent chemoradiotherapy for locally advanced nasopharyngeal carcinoma with nodal stage N2-3. Jpn J Clin Oncol 47:705-712. https:// doi.org/10.1093/jjco/hyx057

21. Hennessy MA, Morris PG (2019) Induction treatment prior to chemoradiotherapy in nasopharyngeal carcinoma. Anti-Cancer Drugs 31:1. https:// doi.org/10.1097/CAD.0000000000000867

\section{Publisher's Note}

Springer Nature remains neutral with regard to jurisdictional claims in published maps and institutional affiliations. 